\title{
The stepwise selection for ketoconazole resistance induces upregulation of C14-demethylase (CYP51) in Leishmania amazonensis
}

\author{
Valter Viana Andrade-Neto', Herbert Leonel de Matos-Guedes², \\ Daniel Cláudio de Oliveira Gomes ${ }^{2,3}$, Marilene Marcuzzo do Canto-Cavalheiro', \\ Bartira Rossi-Bergmann², Eduardo Caio Torres-Santos ${ }^{1 /+}$
}

'Laboratório de Bioquímica de Tripanosomatídeos, Instituto Oswaldo Cruz-Fiocruz, Av. Brasil 4365, 21040-900 Rio de Janeiro, RJ, Brasil
2Instituto de Biofísica Carlos Chagas Filho, Universidade Federal do Rio de Janeiro, Rio de Janeiro, RJ, Brasil
32Departamento de Patologia, Núcleo de Doenças Infecciosas, Universidade Federal do Espírito Santo, Vitória, ES, Brasil

Ketoconazole is a clinically safe antifungal agent that also inhibits the growth of Leishmania spp. A study was undertaken to determine whether Leishmania parasites are prone to becoming resistant to ketoconazole by upregulating C14-demethylase after stepwise pharmacological pressure. Leishmania amazonensis promastigotes [inhibitory concentration $(I C)_{50}=2 \mu \mathrm{M}$ ] were subjected to stepwise selection with ketoconazole and two resistant lines were obtained, La8 $\left(I C_{50}=8 \mu \mathrm{M}\right)$ and La10 $\left(I C_{50}=10 \mu \mathrm{M}\right)$. As a result, we found that the resistance level was directly proportional to the C14-demethylase mRNA expression level; we also observed that expression levels were six and 12 times higher in La8 and La10, respectively. This is the first demonstration that $\mathrm{L}$. amazonensis can up-regulate C14-demethylase in response to drug pressure and this report contributes to the understanding of the mechanisms of parasite resistance.

Key words: drug resistance - azoles - ergosterol biosynthesis

Leishmania parasites are dimorphic protozoa responsible for an endemic disease that affects approximately 12 million people worldwide, with two million new cases occurring annually.

The first choice of treatment against leishmaniasis remains based mainly on the pentavalent antimonials, sodium stibogluconate and N-methylglucamine antimoniate. Alternatively, other drugs, such as pentamidine, non-formulated and lipid-formulated amphotericin B and paromomycin, are used as a second option. Recently, miltefosine, an alkylphosphocholine originally developed as an anticancer drug, was incorporated into the antileishmanial arsenal as the first oral drug effective against visceral leishmaniasis and can also be potentially active against New World cutaneous leishmaniasis (Machado et al. 2010). Nevertheless, all the available drugs present drawbacks that include high toxicity, side effects, drug resistance and/or high cost (Santos et al. 2008).

Imidazole and triazole drugs, such as ketoconazole and itraconazole, have showed to be clinically safe and useful for fungal infections. In addition, they are effective in inhibiting the growth of several Leishmania spp and Trypanosoma cruzi in vitro. Their proposed action mechanism is the blockade of the parasite cytochrome P450-dependent C14-demethylase (CYP51), which causes the accumulation of $14 \alpha$-methylsterols and decreases the production of ergostane-derived sterols in fungi and try-

Financial support: FIOCRUZ

+Corresponding author: ects@ioc.fiocruz.br

Received 30 June 2011

Accepted 7 November 2011 panosomatid parasites (Roberts et al. 2003). Although most Leishmania spp seem to depend on the de novo sterol biosynthesis, promastigotes of Leishmania donovani, Leishmania braziliensis and Leishmania amazonensis are particularly more sensitive to ketoconazole than Leishmania aethiopica, Leishmania major, Leishmania tropica and Leishmania mexicana (Croft et al. 2006). Furthermore, studies also show a perturbation of sterol biosynthesis by ketoconazole in the intracellular amastigotes of L. mexicana (Berman et al. 1986). However, clinical resistance to azoles has been reported in Candida albicans infections, which is particularly threatening in human immunodeficiency virus-infected patients (Rautemaa et al. 2008). Fungal resistance has been shown to be due to drug efflux following the upregulation of $\mathrm{ABC}$ multidrug transporters, as well as the upregulation of several $E R G$ genes that code for enzymes in the sterol biosynthesis pathway (Croft et al. 2006, Tsao et al. 2009). Mutations, rearrangements and amplifications of drug target genes are common events in the selection for drug resistance and gene amplification following the stepwise selection for drug resistance is a well-known phenomenon especially for Leishmania (Coderre et al. 1983). The induction of resistance and upregulation of the sterol C14demethylase expression level have also been described in T. cruzi treated with azoles (Buckner et al. 1998). However, despite the documented development of resistance to drugs, such as pentavalent antimonials (Ashutosh et al. 2007) and paromomycin (Maarouf et al. 1998), nothing is reportedly known about the development of an azole resistance for Leishmania (Croft et al. 2006).

This study was undertaken to determine whether Leishmania parasites are prone to becoming resistant to ketoconazole by upregulating C14-demethylase while under pharmacological pressure. 
To perform resistance studies, L. amazonensis (MHOM/BR/77/LTB 0016 strain) promastigotes [ketoconazole inhibitory concentration (IC) ${ }_{50}=2 \mu \mathrm{M}$ ] were cultured at $26^{\circ} \mathrm{C}$ in Schneider's insect medium (Sigma Aldrich) with increasingly stepwise ketoconazole concentrations, which were supplemented with $10 \%$ heat-inactivated foetal calf serum (HIFCS), $100 \mu \mathrm{g} / \mathrm{mL}$ of streptomycin and $100 \mathrm{U} / \mathrm{mL}$ of penicillin. Ketoconazole solution stock (20 $\mathrm{mM}$ ) was prepared in dimethyl sulfoxide (DMSO) (Sigma-Aldrich). The treated and control cultures received the same solvent concentration, which never exceeded $0.5 \%$ DMSO. The parasites were exposed to drug pressures with an initial ketoconazole concentration of $2 \mu \mathrm{M}$ that was sequentially increased to $4 \mu \mathrm{M}, 8 \mu \mathrm{M}, 10 \mu \mathrm{M}$ and $16 \mu \mathrm{M}$. The cells were microscopically monitored and the passages were performed with initial inoculums of $1 \mathrm{x}$ $10^{6}$ promastigotes $/ \mathrm{mL}$. The drug pressure was maintained throughout the entire study. To determine the level of resistance, the parasites were suspended at $1 \times 10^{6} / \mathrm{mL}$ in Schneider's medium containing $10 \%$ HIFCS and were incubated at $26^{\circ} \mathrm{C}$ in 96 -well, flat-bottom culture plates and in the presence of various concentrations of ketoconazole for $72 \mathrm{~h}$. At the end of incubation, the parasite viability was measured by adding $100 \mu \mathrm{g} / 20 \mu \mathrm{L}$ of 3-(4,5-dimethylthiazol-2-yl)-2,5-diphenyltetrazolium bromide (Molecular Probes, Eugene, USA) and incubating the plates for $2 \mathrm{~h}$ in the dark at $37^{\circ} \mathrm{C}$. Next, dimethylsulphoxide ( 80 $\mu \mathrm{L} /$ well) was added to dissolve the formazan crystals and absorbance was measured in a $\mu$ Quant Microplate Spectrophotometer (Bio Tek Instruments, Winooski, USA). The $\mathrm{IC}_{50}(95 \%$ confidence interval) was determined using the GraphPad Prism software. Resistant lines with $\mathrm{IC}_{50}$ of $8 \mu \mathrm{M}$ and $10 \mu \mathrm{M}$ (denominated La8 and La10) were obtained after 10-12 passages.

To evaluate the correlation of drug resistance with the increased expression of C14-demethylase, a comparative analysis of the enzyme gene expression was conducted by both qualitative and quantitative realtime-polymerase chain reaction (RT-PCR) in the total RNA extracted from wild-type (WT), La8 and La10 promastigotes. The total RNA was extracted from $10^{8}$ L. amazonensis promastigotes (WT, La8 and La10) that were in a logarithmic-growth phase by homogenisation in $1 \mathrm{~mL}$ of Trizol reagent (Invitrogen). DNA contamination was eliminated by treatment with DNAase I (Boehringer Mannheim, Germany) and RNA was quantified using a spectrophotometer. cDNA was synthesised from $5 \mu \mathrm{g}$ of each RNA sample by SuperScript Indirect cDNA labelling Kit (Invitrogen). C14-demethylase expression was estimated by RT-PCR using specific primers (C14forward:GCCGGCCTGGCGCTGTACGGCTGG and C14-reverse: GTACTTCACGCGGCACTGGCTGGCCGT). Primers designed to the actin gene (actin-forward: ATGGCTGACAACGAGCAGAGCTCCA and actinreverse: TCAGAAGCACTTGTTGTGCACGATG) were used as controls. The PCR was performed in a $25 \mu \mathrm{L}$ of reaction volume containing $1 \mathrm{U}$ of Platinum Taq (Invitrogen), Platinum Taq buffer, $1.5 \mathrm{mM} \mathrm{MgCl}, 200 \mu \mathrm{M}$ of deoxynucleoside triphosphates, $5 \%$ of DMSO and 20 pMol of each primer. The amplification program was conducted as follows: $95^{\circ} \mathrm{C}$ for $5 \mathrm{~min}, 35$ repeated cycles of $1 \mathrm{~min}$ at $95^{\circ} \mathrm{C}, 1 \mathrm{~min}$ at $60^{\circ} \mathrm{C}$ (for C14-demethylase) or $55^{\circ} \mathrm{C}$ (for actin) and $2 \mathrm{~min}$ at $72^{\circ} \mathrm{C}$, followed by a single terminal extension at $72^{\circ} \mathrm{C}$ for $15 \mathrm{~min}$. All reactions were performed in an Eppendorf Mastercycler. The PCR products were separated and assessed via electrophoresis in $1 \%$ agarose (Sigma).

To measure the differential expression levels of C14-demethylase in WT and drug-resistant parasites, the extracted RNA was submitted to real-time quantitative PCR using the SYBR Green I technique and the 7500 Real-Time PCR System sequence detection system (Applied Biosystems). Primers for C14-demethylase (forward: GGCCAGCACACCTCCACTAT and reverse: CTTGTTGCGAGGATCCATCA) and actin (forward: ACGCTCCTCGCCACGTT and reverse: TCATGGCCTGCATGTTCTTC) genes were designed using Primer Express ${ }^{\circledR}$ Software v. 2.0. Reactions were performed using SYBR ${ }^{\circledR}$ Green PCR Master Mix and the recommended default conditions on Applied Biosystems RealTime PCR instrument, 40 cycles of denaturation at $95^{\circ} \mathrm{C}$ for $15 \mathrm{~s}$ and annealing extension at $60^{\circ} \mathrm{C}$ for $1 \mathrm{~min}$. Each measurement was performed in triplicate. C14-demethylase gene expression levels relative to actin gene expression levels (endogenous control) for both WT and ketoconazole resistant parasites (La8 and La10) were calculated using the $2^{-\Delta \Delta C}$ method (Livak \& Schmittgen 2001). The CT value for actin varied by approximately one cycle across these three samples; therefore, it was considered to be an appropriate endogenous control. The results were expressed as relative expressions of C14demethylase mRNA levels in resistant compared to WT parasites. The specificity of SYBR Green PCR products was monitored by analysing the amplification profiles using agarose electrophoresis and the corresponding dissociation curves of each amplicon. The amplicons with the expected sizes were observed. Dissociation curves with a single peak were obtained for both C14-demethylase and actin with a melting temperature that varied within $2^{\circ} \mathrm{C}$ of the expected temperature, suggesting an amplification of a specific product (data not shown).

An increase in La8 and La10 C14-demethylase mRNA expression was observed relative to WT parasites (Fig. 1A), which corresponds to the resistance levels of the parasites. The relative quantification of C14demethylase gene in La8 and La10 was showed to be six and 12 times higher, respectively, than that in the WT parasites (Fig. 1B). These results suggest that the increased resistance to ketoconazole is associated with increased C14-demethylase gene expression. Nevertheless, gene regulation in trypanosomatids occurs posttranscriptionally by means of mRNA half-life modulation (D'Orso et al. 2003). Therefore, it would be possible that raised levels of mRNA to C14-demethylase do not reflect raised expression of the protein. Thus, the C14demethylase activity of the resistant lines in comparison to the WT was evaluated. The cell homogenates used for the enzyme assay were obtained by five cycles of freezethaw of $10^{7}$ promastigotes in buffer ( $\left.\mathrm{pH} 7.4\right)$ containing $10 \mathrm{mM}$ HEPES, $400 \mathrm{mM}$ manitol, $10 \mathrm{mM} \mathrm{KCl,} 1 \mathrm{mM}$ magnesium acetate, $1 \mathrm{mM}$ phenylmethanesulfonylfluoride, $10 \mu \mathrm{M}$ E-64 and $1 \mathrm{mM}$ pepstatine. The protein 
A

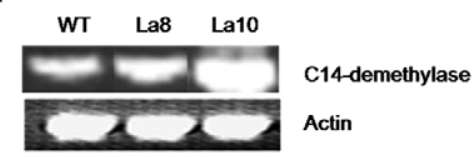

B

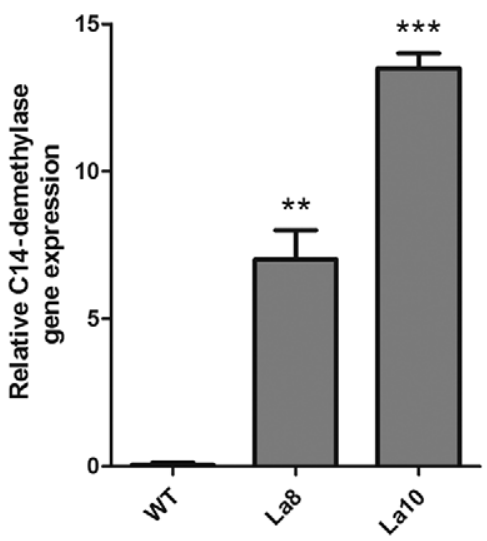

Fig. 1: comparison of C14-demethylase mRNA levels in resistant parasites relative to wild-type (WT). A: WT, La8 and La10 Leishmania amazonensis promastigotes were processed for mRNA extraction for semi-quantitative; B: quantitative real-time-polymerase chain reaction. Relative gene expression $\left(2_{T}^{-\Delta \Delta C}\right) \pm$ standard deviation was calculated using the ${ }^{\Delta \Delta \mathrm{C}}$ method with actin as the endogenous control; **: $\mathrm{p}<0.01 ; * * *: \mathrm{p}<0.001$.

content was determined using the Bradford method and the samples were adjusted to $1 \mathrm{mg} / \mathrm{mL}$. Cell homogenates were incubated with $400 \mu \mathrm{M}$ lanosterol (substrate) and $1 \mathrm{mM}$ nicotinamide adenine dinucleotide phosphate (NADPH). Controls were run without NADPH or lanosterol. The NADPH consumption was unaltered in the absence of lanosterol. C14-demethylase activity was detected spectrophotometrically by measuring NADPH consumption at $390 \mathrm{~nm}$. After $40 \mathrm{~min}$ of incubation, the consumption of NADPH was significantly higher in La8 and La10 strains than in the WT (Fig. 2), suggesting that the higher level of C14-demethylase mRNA is associated with elevated enzyme activity.

The success of leishmaniasis chemotherapy is influenced by many factors, such as host immune status, drug pharmacokinetics, molecular and biochemical differences among the Leishmania species and drug resistance, which is a major cause of treatment failure (Croft et al. 2006).

In this work, we report that the stepwise pressure of L. amazonensis parasites with ketoconazole results in drug resistance that is accompanied by the upregulation of sterol C14-demethylase mRNA expression, which is revealed by quantitative RT-PCR analysis. Ketoconazole-resistant promastigote lines were generated by gradual increases in drug pressure, as previously shown with other anti-leishmanial drugs (Seifert et al. 2003, Coelho et al. 2008). Two ketoconazole-resistant parasite lines were generated in which one resulted in an $\mathrm{IC}_{50}$ of $8 \mu \mathrm{M}$ and the other of $10 \mu \mathrm{M}$, which are respectively four and five times higher than the parenteral line $\left(\mathrm{IC}_{50}\right.$ of $\left.2 \mu \mathrm{M}\right)$. Previously, Buckner et al. (1998) selected for azole resis-

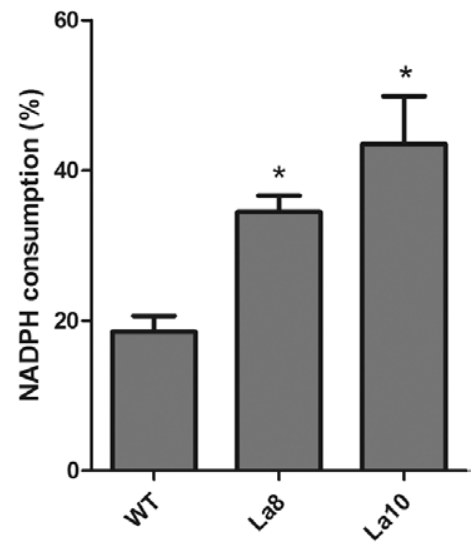

Fig. 2: nicotinamide adenine dinucleotide phosphate (NADPH) consumption associated with C14-demethylase activity. Wild-type (WT), La8 and La10 homogenates were incubated with $400 \mu \mathrm{M}$ lanosterol and $1 \mathrm{mM}$ NADPH for $40 \mathrm{~min}$. The consumption of the co-factor was measured spectrophotometrically at $390 \mathrm{~nm}$. The consumption was expressed as percentage of the optical density in the first minute. Asterisks mean $\mathrm{p}<0.05$.

tance in T. cruzi epimastigotes with itraconazole. More recently, Hankins et al. (2005) have demonstrated that T. cruzi epimastigotes selectively upregulate the sterol C14-demethylase in response to a short exposure to inhibitors of different steps in ergosterol biosynthesis, such as ketoconazole and terbinafine, but not simvastatin. In the present work, we demonstrated that Leishmania parasites can also upregulate the sterol C14-demethylase after drug pressure (Fig. 1). The main molecular mechanisms involved in drug resistance are changes in the uptake, qualitative or quantitative modifications of the targets or the enzymatic inactivation of the drug (Maarouf et al. 1998). The results presented here demonstrate for the first time that $L$. amazonensis can diminish its ketoconazole sensitivity mainly by upregulating the expression of C14-demethylase. However, other mechanisms should not be disregarded, such as qualitative target changes and efflux pumps. Our finding that $L$. amazonensis can upregulate C14-demethylase to compensate for enzyme inactivation contributes to a better understanding of the molecular mechanisms involved in leishmanial resistance to drugs and helps to optimise the use of azoles in the treatment of leishmaniasis.

\section{REFERENCES}

Ashutosh SS, Goyal N 2007. Molecular mechanisms of antimony resistance in Leishmania. J Med Microbiol 56: 143-153.

Berman JD, Goad LJ, Beach DH, Holz GG 1986. Effects of ketoconazole on sterol biosynthesis by Leishmania mexicana mexicana amastigotes in murine macrophage tumor cells. Mol Biochem Parasitol 20: 85-92.

Buckner FS, Wilson AJ, White TC, Van Voorhis WC 1998. Induction of resistance to azole drugs in Trypanosoma cruzi. Antimicrob Agents Chemother 42: 3245-3250.

Coderre JA, Beverley SM, Schimke RT, Santi DV 1983. Overproduction of a bifunctional thymidylate synthetase-dihydrofolate reductase and DNA amplification in methotrexate-resistant Leishmania tropica. Proc Natl Acad Sci USA 80: 2132-2136. 
Coelho AC, Gentil LG, da Silveira JF, Cotrim PC 2008. Characterization of Leishmania (Leishmania) amazonensis promastigotes resistant to pentamidine. Exp Parasitol 120: 98-102.

Croft SL, Sundar S, Fairlamb AH 2006. Drug resistance in leishmaniasis. Clin Microbiol Rev 19: 111-126.

D’Orso I, De Gaudenzi JG, Frasch ACC 2003. RNA-binding proteins and mRNA turnover in trypanosomes. Trends Parasitol 19: $151-155$

Hankins EG, Gillespie JR, Aikenhead K, Buckner FS 2005. Upregulation of sterol C14-demethylase expression in Trypanosoma cruzi treated with sterol biosynthesis inhibitors. Mol Biochem Parasitol 144: 68-75.

Livak KJ, Schmittgen TD 2001. Analysis of relative gene expression data using real-time quantitative PCR and the $2^{-\Delta \Delta C}$ T method. Methods 25: 402-408.

Maarouf M, Adeline MT, Solignac M, Vautrin D, Robert-Gero M 1998. Development and characterization of paromomycin-resistant Leishmania donovani promastigotes. Parasite 5: 167-173.

Machado PR, Ampuero J, Guimarães LH, Villasboas L, Rocha AT, Schriefer A, Sousa RS, Talhari A, Penna G, Carvalho EM 2010. Miltefosine in the treatment of cutaneous leishmaniasis caused by Leishmania braziliensis in Brazil: a randomized and controlled trial. Plos Negl Trop Dis 4: e912.

Rautemaa R, Richardson M, Pfaller M, Perheentupa J, Saxen H 2008. Reduction of fluconazole susceptibility of Candida albicans in APECED patients due to long-term use of ketoconazole and miconazole. Scand J Infect Dis 40: 904-907.

Roberts CW, McLeod R, Rice DW, Ginger M, Chance ML, Goad LJ 2003. Fatty acid and sterol metabolism: potential antimicrobial targets in apicomplexan and trypanosomatid parasitic protozoa. Mol Biochem Parasitol 126: 129-142.

Santos DO, Coutinho CER, Madeira MF, Bottino CG, Vieira RT, Nascimento SB, Bernardino A, Bourguignon SC, Corte-Real S, Pinho RT, Rodrigues CR, Castro HC 2008. Leishmaniasis treatment - a challenge that remains: a review. Parasitol Res 103: 1-10.

Seifert K, Matu S, Perez-Victoria FJ, Castanys S, Gamarro F, Croft SL 2003. Characterisation of Leishmania donovani promastigotes resistant to hexadecylphosphocholine (miltefosine). Int $J$ Antimicrob Agents 22: 380-387.

Tsao S, Rahkhoodaee F, Raymond M 2009. Relative contributions of the Candida albicans ABC transporters CDR1p and CDR2p to clinical azole resistance. Antimicrob Agents Chemother 53: 1344-1352. 